\title{
Cáncer de mama asociado a mutación en genes BRCA-1 y BRCA-2
}

\author{
Breast cancer associated with mutation in the BRCA-1 and BRCA-2 genes
}

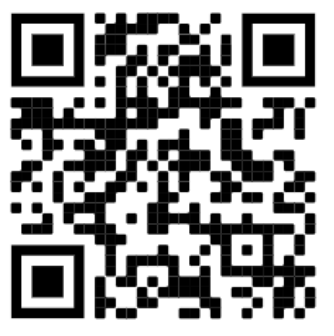

Recibido $10 / 08 / 2020$ Corregido
$02 / 09 / 2020$

Aceptado

$08 / 11 / 2020$

\section{RESUMEN}

El cáncer de mama es el tumor maligno más frecuente en mujeres y la primera causa de muerte en países desarrollados. Su incidencia está en aumento pero su diagnóstico precoz ha logrado disminuir la mortalidad. En algunas ocasiones, el cáncer de mama obedece a mutaciones genéticas heredadas. El tejido tumoral de los cánceres de mama relacionado a mutaciones en la línea germinal requiere un detallado estudio histológico y molecular que determinen las principales características del tumor a fin de establecer riesgo, manejo, pronóstico y sobrevida del paciente. Los genes mayormente implicados a mutaciones en la línea germinal son el BRCA1 y BRCA2. Aquellos tumores asociados a estas mutaciones presentan características tumorales más agresivas en relación a quienes no portan una mutación, lo que se relaciona a un peor pronóstico.

PALABRAS CLAVE: proteína BRCA1, proteína BRCA2, mutación, neoplasias de la mama, histología, fenotipo.

\section{ABSTRACT}

Breast cancer is the most common malignant tumor in women and the leading cause of death in developed countries. Its incidence is increasing but its early diagnosis has managed to reduce mortality. In some cases, breast cancer is due to inherited genetic mutations. The tumor tissue of breast cancers related to mutations in the germ line requires a detailed histological and molecular study that determines

\footnotetext{
${ }^{1}$ Médico general, graduado de la Universidad Internacional de las Américas (UIA). cod. MED16766, correo: chgary04@gmail.com

${ }^{2}$ Médico general, graduado de la Universidad Internacional de las Américas (UIA), cod. MED16764, correo: erick94cr@hotmail.com

${ }^{3}$ Médica general, graduada de la Universidad Internacional de las Américas (UIA), correo: yorlingf95@gmail.com
} 
the main characteristics of the tumor in order to establish risk, management, prognosis and survival of the patient. The genes most implicated with mutations in the germ line are BRCA1 and BRCA2. Those tumors associated with these mutations present more aggressive tumor characteristics in relation to those who do not carry a mutation, which is related to a worse prognosis.

KEYWORDS: BRCA1 protein, BRCA2 protein, mutation, breast neoplasms, histology, phenotype.

\section{INTRODUCCIÓN}

El cáncer de mama es el segundo tipo de cáncer más común y la principal causa de muertes por cáncer en mujeres en todo el mundo. El factor más importante implicado en la aparición de cáncer de mama en mujeres jóvenes es la herencia, específicamente la mutación en los genes de susceptibilidad BRCA1 y BRCA2, los cuales juegan un papel importante como proceso favorecedor en el desarrollo y aparición del cáncer en estas pacientes.

El estudio del cáncer de mama relacionado a mutaciones en los genes BRCA requiere un detallado estudio histológico y molecular del tejido tumoral, debido a que estos aspectos aportan información sobre el origen del tumor $y$ se relacionan estrechamente con el pronóstico y sobrevida del paciente. Generalmente los tumores asociados a mutaciones se caracterizan por un carácter más agresivo.

Los tumores derivados de la mama se categorizan principalmente en 2 aspectos, el primero a nivel histológico donde la mayoría de las neoplasias malignas de mama surgen de elementos epiteliales y se clasifican como carcinomas. Cabe resaltar que los carcinomas de mama son un grupo diverso de lesiones que difieren en apariencia microscópica y comportamiento biológico (1).

El segundo aspecto se enfoca a nivel molecular, donde los tumores de mama se pueden describir en dependencia de los subtipos de expresión génica e inmunohistoquímica. Los subtipos luminales representan los más comunes y se caracterizan por la expresión de receptores de estrógeno (ER) y progesterona (PR); los subtipos basales se incluyen principalmente en la categoría de cáncer de mama triple negativo (TNBC) porque son ER, PR y factor de crecimiento epidérmico del receptor humano 2 (HER2) negativos y representan aquellos de mayor agresividad y peor pronóstico (2).

El objetivo principal de la revisión bibliográfica es establecer las principales características histopatológicas e inmunohistoquímicas del cáncer de mama asociado a mutación en los genes BRCA1 y BRCA2.

\section{MÉTODO}

El artículo está basado en una revisión bibliográfica de 17 fuentes de información publicadas entre los años 2016 y 2020; todos ellos en su idioma original, ya sea en inglés o en español.

Los artículos utilizados proceden de diferentes fuentes digitales entre las que se encuentran Uptodate, Pubmed, Scielo, ELSERVIER y BINASSS. La literatura es procedente de países como Estados Unidos, Cuba, Colombia, Perú, Marruecos y Costa Rica.

Se seleccionaron revisiones basadas en la mutación de los genes BRCA1 y BRCA2, cáncer de mama hereditario, factores de riesgo, epidemiologia y diagnóstico. Se 
evaluaron las publicaciones seleccionadas mediante el análisis del texto completo. El estudio se enfocó en la información más actualizada y en los artículos que presentaran mayor relación con el tema.

\section{EPIDEMIOLOGÍA}

El cáncer de mama es el segundo cáncer con mayor frecuencia diagnosticado en todo el mundo, incluidos los países de ingresos bajos y medianos. Además de su alta prevalencia es un cáncer con altas tasas de mortalidad, en los Estados Unidos representa más de 260,000 casos cada año y es responsable de más de 40,000 muertes aproximadamente ()ㅡ.

En la población occidental, se calcula que alrededor de 5 a $10 \%$ de los pacientes con cáncer de mama pueden portar mutaciones genéticas de susceptibilidad al cáncer hereditario, siendo en el gen BRCA1 la mutación más común. La tasa de mutación puede ser mayor en los judíos asquenazíes. Curiosamente, las tasas de mutación BRCA1 y BRCA 2 en asiáticos son más bajas que en los blancos (무).

Se estima además, que hasta el $25 \%$ del cáncer de mama hereditario se ha relacionado con mutaciones en la línea germinal de genes específicos (4)). Un estudio realizado en el año 2017 reportó que el antecedente patológico familiar de cáncer de mama fue el factor que predominó con una frecuencia de un 56,7 \% entre aquellas pacientes con esta patología (므).

\section{BRCA1 Y BRCA2 COMO FACTORES DE RIESGO EN EL DESARROLLO DEL CÁNCER DE MAMA}

Dentro de los factores de riesgo asociados a mutaciones en la línea germinal, los genes más estudiados son el BRCA1 y BRCA2, cuyas mutaciones altamente penetrantes están asociadas con el de cáncer de mama, representando un rasgo hereditario autosómico dominante que predispone a las mujeres a esta patología. Se estima que aquellos pacientes con mutaciones en los genes BRCA tiene un riesgo de por vida de desarrollar cáncer de mama del $45 \%$ al $75 \%$ aproximadamente (4).

BRCA1 y BRCA2 son genes supresores de tumores involucrados en la reparación del daño del ADN, el control del ciclo celular, la regulación de la transcripción génica y la apoptosis. Las mutaciones comunes de la línea germinal del gen BRCA1 son 5382insC, 185delAG, 3819del5 y 4153delA y de BRCA2 son 4075delGT y 580del4 (ㅁ). Estas mutaciones impiden una adecuada reparación del ADN y generan alteraciones en la función supresora tumoral de estos genes.

Se han observado varias mutaciones fundadoras (mutaciones particulares de los genes BRCA1 y BRCA2 que ocurren entre grupos étnicos definidos o individuos de un área geográfica específica). Además de los judíos asquenazíes, también se han informado mutaciones fundadoras en otras poblaciones, incluyendo europeos, canadienses, hispanos y afroamericanos (므). En la práctica clínica, es difícil proporcionar predicciones altamente individualizadas sobre el riesgo de cáncer de mama asociado a mutaciones. Por lo tanto, a menudo se estudia una variedad de riesgos que pueden contribuir a un incremento en el rango de mutación y luego se adaptan las estimaciones de riesgo al gen implicado (edad actual, antecedentes de cáncer de mama y uso de terapia hormonal) $(\underline{5}, \underline{6})$.

En relación a los antecedentes de cáncer de mama como factor de riesgo asociado a mutación, un análisis agrupado de más de 50,000 mujeres con cáncer de mama y 
100,000 controles, determinó que el riesgo aumenta casi el doble si una mujer tiene un familiar de primer grado afectado y además se triplicó si tenía dos parientes afectados de primer grado (ㅁ).

Las variaciones genéticas comunes, llamadas polimorfismos de un solo nucleótido, también pueden contribuir significativamente al riesgo, particularmente en los portadores de mutación en el gen BRCA2; sin embargo, estos generalmente no se evalúan en la práctica clínica habitual. En general, el rango de riesgo de cáncer de mama en portadores de mutación en el gen BRCA1 es de entre 55 y $70 \%$ hasta los 70 años de edad y del 45 a $70 \%$ en BRCA2 (ㅁ)).

\section{FENOTIPO HISTOLÓGICO E INMUNOHISTOQUÍMICA DEL CÁNCER DE MAMA}

Desde el aspecto histológico, los tumores de mama se clasifican según la afección o no de la membrana basal, estos son los no invasivos (in situ) o invasivos. Dentro de los carcinomas no invasivos se encuentra el carcinoma intraductal in situ y el carcinoma lobulillar in situ. Los carcinomas de mama invasivos consisten en varios subtipos histológicos; siendo el más frecuente el tipo ductal infiltrante en un $76 \%$, seguido por el lobular invasivo en un $8 \%$, ductal/lobular $(7 \%)$, mucinoso $(2,4 \%)$, tubular $(1,5 \%)$ y medular $(1,2 \%)$ (1).

La otra manera de clasificar el cáncer de mama es mediante la inmunohistoquímica, que permite detectar proteínas en las células, lo cual ha llevado a categorizar los carcinomas de mama según la expresión de ER, PR, HER2 y el índice de proliferación celular ki-67 (ㅇ).

Entre el $75 \%$ y el $80 \%$ de los cánceres son positivos para receptores hormonales de ER o PR, y entre el $15 \%$ y el $20 \%$ son positivos para HER2. El porcentaje remanente entre el $10 \%$ y el $15 \%$ representa los TNBC definidos por la ausencia de expresión de receptores hormonales y de HER2 (ㅇ).

Se han identificado cuatro subtipos moleculares de cáncer de mama: luminal $A$, luminal B, HER2 y tipo basal, mediante la aplicación de los perfiles de expresión génica, que es la mejor forma de clasificar los carcinomas de mama ( $\underline{9})$.

Cabe resaltar que el fenotipo Luminal $A$ es aquel con ER (+) y/o PR (+); HER2 (-), siendo el subtipo más común y menos agresivo. El luminal B tiene ER (+) y/o PR $(+)$; HER2 (+), con características similares al luminal A pero con peores resultados. El basal like o triple negativo, es aquel con ER (-); PR (-); HER2 (-) CK 5/6 (+) y/o receptor del factor de crecimiento epidérmico (EGFR) $(+)$, siendo el más agresivo, con alto grado histológico y el de peor pronóstico, por último el HER2 (+); ER (-) que es menos común pero altamente agresivo (10).

El Ki 67 demuestra el índice de proliferación celular, determinando $20 \%$ como el límite para establecer que si es $<20 \%$ con receptores hormonales positivos será Luminal A y si es $>20 \%$ entonces, se considera Luminal $\mathrm{B}$.

Hasta el $20 \%$ de los pacientes con TNBC albergan una mutación del gen BRCA, particularmente en BRCA1. Por el contrario, menos del $6 \%$ de todos los cánceres de seno están asociados con una mutación BRCA. Ante este hallazgo, cualquier paciente con enfermedad triple negativa debe ser derivado a un asesor genético para analizar las pruebas de línea germinal BRCA. Además, cualquier paciente de 60 años o menos con TNBC debe someterse a una prueba de línea germinal BRCA (11). Además de la histopatología e inmunohistoquímica, una 
caracterización completa del tumor requiere conocer aspectos como el tamaño tumoral, el compromiso ganglionar y el grado de diferenciación. El tamaño del tumor $(T)$, se define como el mayor diámetro del tumor de mama primario, se reconoció pronto como un factor pronóstico importante en el cáncer de mama. El tamaño del tumor se correlaciona con la afectación ganglionar, pero el valor pronóstico de los dos factores es independiente (12).

Con relación al compromiso ganglionar (número de ganglios axilares ipsilaterales con crecimiento tumoral metastásico), este se considera un factor pronóstico negativo fuerte e independiente. Entre las mujeres sin evidencia de enfermedad metastásica, la tasa de supervivencia a cinco años para aquellas que se presentan con enfermedad localizada (es decir, solo de mama) versus regional (es decir, afectación de ganglios patológicos) es del 99 y $85 \%$, respectivamente. Incluso los tumores pequeños $(<2 \mathrm{~cm})$ tienen un peor pronóstico en presencia de afectación ganglionar patológica (12).

El grado de diferenciación del cáncer de mama se asigna mediante el sistema de clasificación Elston-Ellis, que establece el porcentaje de formación de túbulos, pleomorfismo nuclear y actividad mitótica (12).

En el grado 1 o bien diferenciado las células son de crecimiento más lento y se parecen más al tejido mamario normal.

En el grado 2 o moderadamente diferenciado la velocidad de crecimiento de las células y su aspecto corresponden a un valor entre los grados 1 y 3 .

En el grado 3 o pobremente diferenciado las células cancerosas se ven muy diferentes a las células normales y probablemente crecerán y se propagarán más rápido.

\section{MUTACIÓN EN LOS GENES BRCA1 Y BRCA2 ASOCIADOS A LA INCIDENCIA DE CÁNCER DE MAMA}

Con el advenimiento de las pruebas genéticas en la práctica oncológica, se logró detectar la susceptibilidad de familias de alto riesgo. En la actualidad se han identificado las mutaciones en los genes BRCA1 y BRCA2 como las mutaciones con mayor implicación en el desarrollo de esta patología. De igual forma, se han encontrado otros genes a los que se les ha adjudicado la particularidad de ser predisponentes para el cáncer de mama, sin embargo, su participación no tiene tanta significancia estadística en la patogenia del cáncer de mama.

Una cohorte prospectiva de 252 pacientes con cáncer de mama esporádico no relacionado de la población mexicanamestiza encontró 28 mutaciones patogénicas (25 en BRCA1 y 13 en BRCA2), 11 de los cuales no habían sido reportados previamente en poblaciones hispanas 0 latinoamericanas. Un total de 38 pacientes fueron positivas para una mutación patógena que representa el $15 \%$ de la cohorte de mujeres mexicanas con cáncer de mama; 25 para BRCA1; y 13 para BRCA2 (13).

Un artículo publicado en el año 2016, basado en un meta análisis que recopiló información por medio de una búsqueda literaria de 60 artículos, reportó la incidencia del cáncer de mama relacionado a mutación en los genes BRCA. En general, mostró una relación en donde de los 105,220 pacientes con cáncer de mama que estaban disponibles dentro de los estudios incluidos en el meta análisis, 3588 (3.4\%) fueron portadores de mutaciones BRCA. De estos resultados se indica que 2.263 (63.7\%) de los pacientes 
presentaron mutación en BRCA1, 774 $(21,65 \%)$ en BRCA2 y los restantes 551 $(15,35 \%)$ en ambos genes (ㄴ).

En relación a la incidencia, un artículo colombiano publicado en el año 2017 seleccionó a 853 pacientes del género femenino con cáncer de mama diagnosticadas a cualquier edad en un periodo de seis años (entre 2009-2014) con el fin de evaluar cuales pacientes eran portadoras de alguna mutación en los genes BRCA. Este estudio detectó 107 pacientes portando mutaciones en estos genes, de las cuales 69 (64.5\%) fueron localizadas en BRCA1, y 38 (35.5\%) en BRCA2 (14).

\section{RELACIÓN DE LA MUTACIÓN EN LOS GENES BRCA1 Y BRCA2 CON LA HISTOPATOLOGÍA DEL CÁNCER DE MAMA Y SU DIAGNÓSTICO MOLECULAR}

La biología molecular, ha detectado entre otras explicaciones, la inestabilidad genética y diversas mutaciones en genes supresores tumorales.

Dentro de este grupo de genes están los denominados BRCA1 y BRCA2 los cuales están asociados a una mayor susceptibilidad a desarrollar cáncer de mama hereditarios en por lo menos $50 \%$ de los casos correspondientes. Un estudio publicado en el año 2016 evaluó las características clínicas y patológicas de los tumores BRCA1 y BRCA2 en una muestra de alto riesgo en 40 pacientes marroquíes con cáncer de mama y fuertes antecedentes familiares de esta patología.

Los resultados de secuenciación reportaron que 11 pacientes portaban genes mutados en BRCA1 y BRCA2, y que 29 mujeres no asociaron mutaciones en estos genes.
Esto incluye 6 pacientes con la mutación en el gen BRCA1 y 5 pacientes con mutación BRCA2 (15).

Histológicamente, el carcinoma ductal infiltrante fue el tipo más común en ambos grupos (90.9 y 93.3\%). Los tamaños de tumores T1 y T2 se observaron en pacientes mutados con una frecuencia de $72.7 \%$. El grado III se encontró en $63.6 \%$ de mujeres con mutación BRCA1 y BRCA2 contra una frecuencia de $20.7 \%$ entre los no portadores. Los portadores BRCA1 y BRCA2 tenían más probabilidades de tener cáncer de mama triple negativo comparado con los no portadores (62.5 vs.16.7\%) (15).

Por otra parte, la afectación de ganglios linfáticos, expresión de receptores hormonales y el estado de Her2 no mostró significativa diferencia estadística entre ambos grupos estudiados (15).

En resumen, tal estudio marroquí comparó las características clínicas y patológicas de las mujeres que llevan o no mutación BRCA1 y BRCA2. Los pacientes con mutación BRCA tienden a desarrollar cáncer de mama temprano con alto grado histológico y triple negativo, por ende son más agresivos.

Otro artículo publicado en el año 2017 menciona dentro de su estudio que entre los 1.009 pacientes diagnosticados con cáncer de mama triple negativo, 69 pacientes (6.9\%) tenían mutaciones BRCA1 y 32 pacientes (3.2\%) tenían mutaciones BRCA2. Además, 113 pacientes con diagnóstico triple negativo tenían antecedentes familiares positivos de cáncer de mama y/o de ovario entre los 1.009 pacientes (16).

Dentro del mismo artículo se menciona que de las pacientes examinadas, aquellas que presentaban carcinomas de mama con mutación BRCA2 tenían más probabilidades de ser ER o PR positivos, lo que sugiere que el cáncer de mama asociado a BRCA2 
incluye una proporción relativamente mayor del subtipo luminal (16).

En el estudio se determinó que la expresión de HER2 fue mucho menor en los pacientes con mutación BRCA1 y BRCA2 que en los no portadores. El índice Ki67 (proliferación celular) y los grados tumorales de los cánceres mutados en BRCA1 fueron más altos que los de los cánceres BRCA negativos. Los pacientes con mutación BRCA1 también presentaron una etapa más alta en el momento del diagnóstico. Los portadores de la mutación BRCA2 mostraron más ganglios linfáticos positivos (16).

Un artículo publicado en el año 2016 incluyó como población de estudio 50 pacientes diagnosticadas con cáncer de mama poseedoras de mutaciones de los genes BRCA (19 con mutaciones de BRCA1 y 31 en BRCA2), estos fueron establecidos como el grupo de casos, así como 100 pacientes con cáncer de mama sin mutaciones BRCA conocidas que fueron tomados como el grupo de controles (17).

La media de edad de los casos fue similar a la de los controles, sin diferencias significativas entre los portadores de la mutación BRCA1 y BRCA2. Los tamaños de los tumores también fueron similares entre los casos y los controles (1,4 cm frente a 1,2 $\mathrm{cm})$ respectivamente y entre los portadores de la mutación BRCA1 y BRCA2 (17).

Con base en el estudio, el $90 \%$ de los casos y el $87 \%$ de los controles tenían tumores con histología ductal invasiva. La mediana de la positividad del receptor de estrógenos fue del $90 \%$ para los portadores de BRCA1 y BRCA2, del $95 \%$ para los controles. La expresión media del receptor de progesterona fue del $60 \%$ para los casos y del $85 \%$ para los controles. Los casos fueron significativamente más propensos a tener cánceres histológicos de grado 3. Todos los pacientes tenían enfermedad ganglionar negativa, con la excepción de 1 paciente con una mutación BRCA2 (17).

\section{CONCLUSIONES}

Existe una relación clara entre las características histológicas e inmunohistoquímicas más importantes del cáncer de mama, como la expresión hormonal y el fenotipo, en aquellos casos de pacientes con cáncer de mama portadoras de una mutación en los genes BRCA.

En cuanto a las características histopatológicas del cáncer de mama, los pacientes con mutación en los genes BRCA mostraron que el carcinoma de origen ductal infiltrante o invasivo se asocia en mayor medida.

Comparando las características clínicas y patológicas de las mujeres que llevan o no mutación en los genes BRCA1 y BRCA2, aquellas que portan una mutación BRCA tienden a desarrollar cáncer de mama a edades más tempranas y con alto grado histológico. Aquellos tumores relacionados específicamente al gen BRCA1 presentan un grado histológico alto y fenotipo triple negativo, mientras que los tumores asociados a mutación en el gen BRCA2 asocian más un fenotipo luminal y son en promedio de un grado histológico más alto en comparación con aquellos no asociados a una mutación.

En general, los principales rasgos de los tumores de mama asociados con mutación en los genes BRCA1 y BRCA2 presentan características tumorales más agresivas en relación con quienes no portan una mutación, lo que se relaciona a un peor pronóstico. 


\section{REFERENCIAS}

1. Bleiweiss, I. Pathology of breast cancer. Uptodate, [online]. 2020; 1. Available at: https://www.uptodate.com/contents/pathology-of-breast-cancer

2. Joe, B. Clinical features, diagnosis, and staging of newly diagnosed breast cancer. Uptodate, [online]. 2020; 1. Available at: https://www.uptodate.com/contents/clinical-features-diagnosis-and-staging-of-newly-diagnosedbreast-cancer

3. Pogoda, K., Niwińska, A., Sarnowska, E., Nowakowska, D., Jagiełło-Gruszfeld, A., Siedlecki, J. and Nowecki, Z. Effects of BRCA Germline Mutations on Triple-Negative Breast Cancer Prognosis. Journal of Oncology. 2020; 2. https://doi.org/10.1155/2020/8545643

4. Baretta, Z., Mocellin, S., Goldin, E., Olopade, O. and Huo, D. Effect of BRCA germline mutations on breast cancer prognosis. Medicine. 2016; 95(40): 1. https://doi.org/10.1097/MD.0000000000004975

5. Peña, Y., González, M., Céspedes, Á., Velázquez, L. and López, Y. Factores de riesgo para padecer cáncer de mama en la población femenina. Revista Finlay, [online]. 2017; 287. Available at: http://scielo.sld.cu/pdf/rf/v7n4/rf08407.pdf

6. Isaacs, C. and Peshkin, B. Cancer risks and management of BRCA1/2 carriers without cancer. Uptodate, [online]. 2020; 2-4. Available at: https://www.uptodate.com/contents/cancer-risks-and-management-of-brca1-2-carrierswithout-cancer

7. Chen, W. Patient education: Factors that modify breast cancer risk in women (Beyond the Basics). Uptodate, [online]. 2020; 8, 9. Available at: https://www.uptodate.com/contents/factors-that-modify-breast-cancer-risk-inwomen-beyond-the-basics

8. Espinosa, M. Cáncer de mama. Revista médica Sinergia, [online]. 2018; 9. Available at: https://www.medigraphic.com/pdfs/sinergia/rms-2017/rms171b.pdf

9. Medina, G. Clinical and prognostic characteristics of the molecular subtypes of breast cancer determined by immunohistochemistry. Arequipa, Peru. Revista Perú médica, [online]. 2017; 34: 472. Available at: http://www.scielo.org.pe/scielo.php?script=sci arttext\&pid=S1726-46342017000300014

10. Madrigal, A. and Mora, B. Generalidades de cáncer de mama para médico general. Medicina Legal de Costa Rica Edición Virtual, [online]. 2018; 35: 73. Available at: https://www.scielo.sa.cr/pdf/mlcr/v35n1/1409-0015-mlcr-35$\underline{01-44 . p d f}$

11. Anders, C. and Carey, L. ER/PR negative, HER2-negative (triple-negative) breast cancer. Uptodate, [online]. 2020; 1. https://doi.org/10.1142/9789813277762 0001

12. Foukakis, T. and Bergh, J. Prognostic and predictive factors in early, non-metastatic breast cancer. Uptodate, [online]. 2020; 3. Available at: https://www.uptodate.com/contents/prognostic-and-predictive-factors-in-early-nonmetastatic-breast-cancer

13. Millan Catalan, O., Campos-Parra, A., Vázquez-Romo, R., Cantú de León, D., Jacobo-Herrera, N., MoralesGonzález, F., López-Camarillo, C., Rodríguez-Dorantes, M., López-Urrutia, E. and Pérez-Plasencia, C. A MultiCenter Study of BRCA1 and BRCA2 Germline Mutations in Mexican-Mestizo Breast Cancer Families Reveals Mutations Unreported in Latin American Population. Cancers, [online]. 2019; 11(9): 1246. https://doi.org/10.3390/cancers11091246 
14. Briceño, I., Gómez, A., Díaz, N., Noguera, M., Díaz, D. and Casas, M. Mutational spectrum in breast cancer associated BRCA1 and BRCA2 genes in Colombia. Revista Colombia médica, [online]. 2017 ; 48 : 58. https://doi.org/10.25100/cm.v48i2.1867

15. Jouhadi, H., Tazzite, A., Azeddoug, H., Naim, A., Nadifi, S. and Benider, A. Clinical and pathological features of BRCA1/2 tumors in a sample of high-risk Moroccan breast cancer patients. BMC Research Notes, [online]. 2016; 9: 2, 4. https://doi.org/10.1186/s13104-016-2057-8

16. Lang, G., Xiu, J., Hu, X., Zhang, H., Shan, L. and Song, C. The spectrum of BRCA mutations and characteristics of BRCA-associated breast cancers in China: Screening of 2,991 patients and 1,043 controls by next-generation sequencing. Pubmed. 2017; $\quad 130-136 . \quad$ Available at: http://file://C:/Users/User/Documents/Articulo\%20Ca\%20mama/The\%20Spectrum\%20of\%20BRCA\%20Mutatio ns\%20China.pdf

17. Shah, P., Patil, S., Dickler, M., Offit, K., Hudis, C. and Robson, M. Twenty-one-gene recurrence score assay inBRCA-associated versus sporadic breast cancers: Differences based on germline mutation status. Cancer, [online]. 2016; 122(8): 1180. https://doi.org/10.1002/cncr.29903 